\title{
Machine learning and Data Mining Techniques in Health Monitoring of Artificial Satellites Mission
}

\author{
Ashraf Darwish* \\ Faculty of Science, Egypt \\ *Corresponding author: Ashraf Darwish, Faculty of Science, Egypt
}

Submission:

\section{Editorial}

The development of an intelligent satellite health monitoring system is an essential issue in aerospace engineering that determines the state of health and failure of satellites based on telemetry data. The recent development of data mining and machine learning techniques makes it possible to use satellite telemetry data and to extract integrated information for the production of advanced health monitoring system. The editorial paper can be used as a guideline for the design of an intelligent health monitoring solution based on remote data. Satellites are amongst today's most complex technical systems, they fulfill their mission in a very harsh and special environment. So, it is practically impossible to completely eliminate the possibility of anomalies or faults. Determining the state of health of these systems using traditional know ledge-based techniques or situation-based diagnosis is becoming more difficult due to the diversity of external factors and the degradation of performance, such as the state of the satellite that will change for the design stage, especially long-lived satellites. Therefore, telemetry data mining techniques have been developed to address the health monitoring operations. By analyzing system operations data to characterize and detect unusual behavior or anomalies and analyzing the fault detection and the impact on the system. Then, followed by discovering the relationship between dependent and independent variables to predict the next event or behavior of the satellites that ensures the highest level of reliability and security.

All previous processes will lead to the proper control of satellite based on the status of resources and mission operations. Several health monitoring approaches have been developed and successfully applied to aerospace operations for both real-time systems monitoring and archived data analysis. This study is focused on the main operations of health monitoring, detection, diagnosis, and prediction. In recent years, health monitoring operations based on satellite telemetry data mining have practical significance and have become the research focus of the field of aerospace, many approaches and tools have been recently developed and applied to different satellite missions, the noteworthy examples of these works are presented through an integrated framework with data mining methods. This study presents the state-of-art of the intelligent health monitoring operations and applications.
Telemetry is the collection of measurements and onboard instrument readings required to deduce the health and status of all of the satellite subsystems in the spacecraft bus and the pay load. The satellite telemetry tracking and control subsystem must collect process and transfer the data from the satellite to Earth. Health monitoring of artificial satellites is concerned with the health of the satellite to detect any abnormal behavior. During the artificial satellite operational life time the ground station is receiving the telemetry data, is non-stationary time series dataset contains thousands of sensor measurements from various subsystems, which contains the wealth information related to the health and status of the entire satellite and all its subsystems which reflect the operational status and payload of satellites. The health and status measurements of the satellite include the status of resources, the health and mode of operation for each subsystem and environmental data like values of sun and radiation or like star trackers. The telemetry data is analyzed in the ground control station for the health monitoring purposes such as failure diagnostic or prognostic and anomaly detection.

Telemetry data is non-stationary time series data contains thousands of sensor outputs from multiple different sub systems and each one of these subsystems brings up to thousands of records every day represent health, status, and mode of each one, besides the thousands of the environmental changes and attitude of the satellite measurements. Telemetry data is a heterogeneity and multi-modality data, since it is being composed of hundreds to thousands of variables and attributes, are collected from various sensors, and each one has different output form. Also, each one of the satellite subsystems has different modes of operations to be changed from a specific mode to another one over the time.

Artificial satellites monitoring process requires guarantees to ensure reliable operations of satellites. Therefore, anomaly detection systems have the capability to detect any abnormal behaviors of the satellite subsystems. In the literature, the proposed anomaly detection systems suffer from the variety of faults and their sensitivity. To overcome these problems, the fault detection system is integrated. A new diagnostic-tree method has been presented to improve the fault detection mechanisms 
by classifying the different types of faults. An artificial Satellite's health monitoring is controlled by analyzing the telemetry data which can be received from the satellite at the ground station. This data can be received during the mission process of the Satellite. Therefore, a huge amount of this data can be collected for further analysis. In the last decade, the machine learning and data mining techniques are used to analyze this telemetry data for fault detection, recovery, and prediction. Recently, NASA developed new project in the space missions based on data mining tool called ORICA and Inductive Monitoring System (IMS). ORCA can search for outliers and unusual data points in multivariate data sets by calculating the distance of each data point from neighboring points. On the other hand, the IMS tool is based on the concept of clustering techniques to extract to extract model of normal system about the normal operation for health monitoring process.

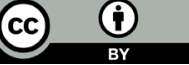

Creative Commons Attribution 4.0 International License

For possible submissions Click Here

\section{Submit Article}

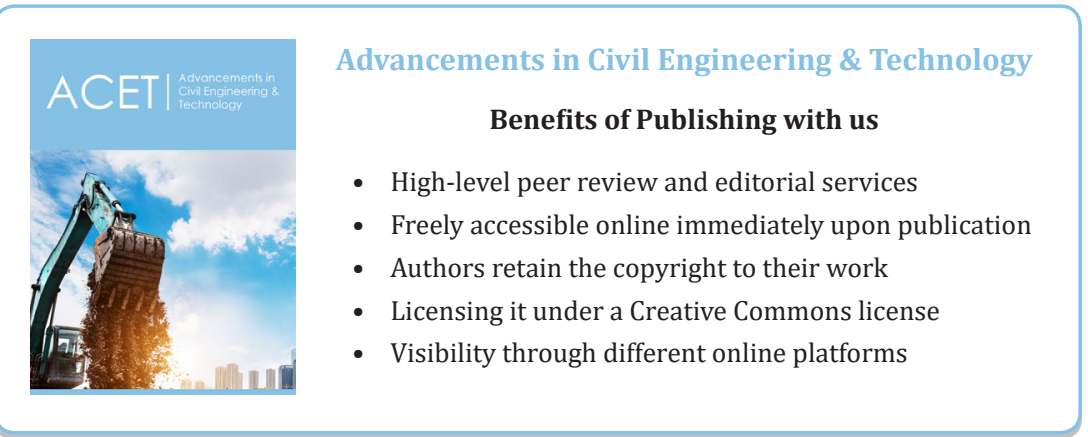

